\title{
Piperine production by endophytic fungus Periconia sp. isolated from Piper longum L.
}

\author{
Vijay C Verma ${ }^{1,5}$, Emil Lobkovsky ${ }^{2}$, Alan C Gange ${ }^{3}$, Santosh K Singh ${ }^{1}$ and Satya Prakash ${ }^{4}$ \\ The endophytic fungus Periconia sp. produces piperine (5-(3, 4-methylenedioxyphenyl)-1-piperidinopent-2, 4-dien-1-one) under \\ liquid culture. This is the first report of the alternative source for this chemical other than its host, Piper longum. The highly \\ functionalized fungus-derived piperine exhibits strong antimycobacterial activity against Mycobacterium tuberculosis and \\ M. smegmetis with minimum inhibitory concentrations of 1.74 and $2.62 \mu \mathrm{g} \mathrm{ml}^{-1}$, respectively. The compound was crystallized \\ and the structure was elucidated by single-crystal X-ray crystallography. This finding is of significance as piperine is a potential \\ cancer preventative agent. It is reaffirmed by this report that important pharmaceuticals can be produced by endophytic \\ microbes, and these molecules appear to be mimetic to their host origin. Therefore, we can enhance the bioactive principles \\ of medicinal plants by isolating and identifying the endophytes, thereby showing the importance of preserving the biodiversity \\ of these plants.
}

The Journal of Antibiotics (2011) 64, 427-431; doi:10.1038/ja.2011.27; published online 20 April 2011

Keywords: antimycobacterial activity; endophytic fungi; Periconia sp.; piperine; X-ray crystallography

\section{INTRODUCTION}

With the discovery of taxol from the endophytic fungus Taxomyces andreanae in pacific yew (Taxus brevifolia) by Stierle et al., ${ }^{1}$ a new era of research in endophytic biology was opened. Until that time, leaf material of the pacific yew was the only source of 'Taxol' known. Since then, other authors have established that certain endophytes can produce an array of chemicals similar to those found in their hosts. ${ }^{2}$ This has encouraged researchers to select medicinal plants for endophytic study that have a known therapeutic as well as ethanobotanical history and that contain a well-characterized set of chemicals. Some well-known systems are Hypericum perforatum-Hypericin, Catharanthus roseus-vincristine/vinblastine and Camptotheca foetida-camptothecin, etc. ${ }^{3-6}$ In the light of these works, we have made efforts to isolate endophytic fungi and bacteria from well-known spice pepper (Piper longum L.), which contains a very potent alkaloid 'piperine'. The dried unripe fruit contains piperine (5-9\%), volatile oil (1-2.5\%), pungent resin (6.0\%), piperidine and starch (about 30\%). The volatile oil contains mainly l-phyllandrene and caryophyllene. Piperine content is 3-5\% (on dry weight basis) in P. longum and varies within different species of piper plant. Piperine is a piperidine derivative with multiple pharmacological and physiological activities. ${ }^{7-8}$ The traditional uses include analgesic, antipyretic, antidepressant, ${ }^{9}$ neuroprotective, ${ }^{10,11}$ anti-inflammatory, ${ }^{12,13}$ antioxidant, ${ }^{14,15}$ anticonvulsant, antibacterial, antitumor ${ }^{16}$ and hepatoprotective activities. ${ }^{17-19}$ Piperine provides protection against seizures in epilepsy ${ }^{20}$ and has been shown to enhance the bioavailability of several drugs, such as sulfadiazine, tetracycline, streptomycin, ${ }^{21}$ rifampicin, pyrazinamide, isoniazid, ${ }^{22}$ ethambutol ${ }^{23}$ and phenytoin. ${ }^{24}$ The pepper plant has formed the basis of many traditional formulations that have been in existence for thousands of years in the Indian system of medicine called 'Ayurveda'. The pepper and its phyto-constituents have an essential role in healthcare in the several other traditional systems of medicine in many other countries. ${ }^{7}$ Thus, given the importance of piperine and other related alkaloids, we attempted to isolate endophytic fungi from $P$. longum plants with the objective to screen and isolate strains that have the potential to produce piperine and related alkaloids as an alternative source other than their host. To date, a large number of bioactive compounds from endophytic microbes in an array of plants have been extracted, isolated and characterized in details with extensive references in several review articles. ${ }^{25-30}$ However, very few reports on endophytic fungi concern P. longum itself and these mainly focus on the potential of some endophytic bacterial strains to show activity against phytopathogens; such as Phytophthora capsici. ${ }^{31}$ The only report other than the present one is the isolation and characterization of piperine from an Ulocladium sp., ${ }^{32}$ but this report does not give details about the biology and ecology of the fungus.

Here, we report for the first time that an endophytic Periconia sp. isolated from P. longum is able to produce piperine and as a result, the fungus has antimycobacterial activity.

${ }^{1}$ Centre of Experimental Medicine and Surgery, Institute of Medical Sciences, Banaras Hindu University, Varanasi, India; ${ }^{2}$ Department of Chemistry, Baker Laboratory, Cornell University, Ithaca, NY, USA; ${ }^{3}$ School of Biological Sciences, Royal Holloway University of London, Surrey, UK and ${ }^{4}$ National Facility for Tribal and Herbal Medicine, Institute of Medical Sciences, Banaras Hindu University, Varanasi, India

${ }^{5}$ Current address: Institut für Umweltforschung (INFU), Technische Universität Dortmund, Otto-Hahn-Str. 6, Campus Nord, 44227 Dortmund, Germany.

Correspondence: Dr VC Verma, Centre of Experimental Medicine and Surgery, Institute of Medical Sciences, Banaras Hindu University, Varanasi, Uttar Pradesh 221005, India. E-mail: chandravcv@gmail.com

Received 2 December 2010; revised 28 February 2011; accepted 8 March 2011; published online 20 April 2011 


\section{MATERIALS AND METHODS}

\section{Plant samples}

A random sample of 10 P. longum L. plants were taken from the campus of Banaras Hindu University, Varanasi $\left(25.5^{\circ} \mathrm{N} 82.9^{\circ}\right.$ E, elevation $279 \mathrm{ft}$ per $\left.85 \mathrm{~m}\right)$ in December 2009. Fresh plant samples of healthy and symptomless leaves, stem and root were collected. The cut ends were wrapped with parafilm before they were placed into sterile polythene bags for further isolation procedures in the laboratory.

\section{Surface treatment, isolation and culture conditions}

A total of 45 tissue segments, 5 from each of 3 leaf, stem and root samples from each of 10 selected plants were taken and surface treated by immersion in $70 \%$ ethanol for $1 \mathrm{~min}, 5 \% \mathrm{NaOCl}$ for $5 \mathrm{~min}, 96 \%$ ethanol for $0.5 \mathrm{~min}$, followed by three rinses in sterile distilled water. All sample tissues were then placed onto separate petri dishes $(60 \mathrm{~mm})$ containing potato dextrose agar supplemented with $250 \mathrm{mg} \mathrm{l}^{-1}$ oxytetracycline hydrochloride (Terramycin, Pfizer, Mumbai, India) for isolating endophytic fungi, ${ }^{33} \mathrm{~S}$-agar medium (containing per litre: $10 \mathrm{~g}$ dextrose, $4.0 \mathrm{~g}$ casein hydrolysate, $0.5 \mathrm{~g} \mathrm{~K}_{2} \mathrm{HPO}_{4}, 0.2 \mathrm{~g} \mathrm{MgSO}_{4} .7 \mathrm{H}_{2} \mathrm{O}, 0.1 \mathrm{~g} \mathrm{CaCl}_{2} .2 \mathrm{H}_{2} \mathrm{O}$, $10 \mathrm{mg}$ ferric citrate, $0.01 \mathrm{mg} \mathrm{CoSO}_{4} .7 \mathrm{H}_{2} \mathrm{O}, 0.1 \mathrm{mg} \mathrm{CuSO}_{4} .5 \mathrm{H}_{2} \mathrm{O}, 1.5 \mathrm{mg} \mathrm{H}_{3} \mathrm{BO}_{3}$, $0.8 \mathrm{mg} \mathrm{MnSO} \mathrm{M}_{4} \cdot \mathrm{H}_{2} \mathrm{O}, 0.2 \mathrm{mg}\left(\mathrm{NH}_{4}\right)_{6} \mathrm{Mo}_{7} \mathrm{O}_{24} \cdot 4 \mathrm{H}_{2} \mathrm{O}, 0.6 \mathrm{mg} \mathrm{ZnSO} \cdot .7 \mathrm{H}_{2} \mathrm{O}$ and $6.0 \mathrm{~g}$ agar) for isolating actinomycetes ${ }^{34}$ and nutrient agar for isolating endophytic bacteria. All petri dishes were sealed with sterile parafilm to protect them from contamination during repeated handling while examining endophytes, and from desiccation. The plates were incubated at $26 \pm 2{ }^{\circ} \mathrm{C}$ and $98 \%$ relative humidity (under $12 \mathrm{~h}$ fluorescent light $/ 12 \mathrm{~h}$ dark light), enclosed in translucent white-cover plastic boxes, in a biological oxgen demand cum humidity incubator (Remi, Mumbai, India; VDF-265) for 25 days. Fungi that grew from the tissue fragments were subcultured onto fresh potato dextrose agar plates. Isolates that failed to produce reproductive structures were subcultured onto $2 \%$ malt yeast extract (Oxoid, Mumbai, India; malt extract $\mathrm{L}_{39} 20 \mathrm{gl}^{-1}$, and agar $20 \mathrm{gl}^{-1}$ ) supplemented with streptomycin $250 \mathrm{mgl}^{-1}$ in a petri dish $(50 \mathrm{~mm})$ containing a thin strip of autoclaved host tissue to encourage the sporulation. Cultures that failed to sporulate even under these conditions were considered sterile.

\section{Identification of endophytic Periconia sp.}

All microscopic analysis was on the basis of observations with an eclipse E600 research microscope (Nikon, Tokyo, Japan) attached with coolpix 3.34 MP digital camera. Scanning electron microscopy (SEM) was performed on Periconia sp. using the techniques described earlier by Ezra $e t$ al..$^{35}$ The fungal biomass was dried and a gold coating was done using sputtering technique and examined with a JEOL6100 SEM (JEOL Ltd., Tokyo, Japan). However, because the conidia and conidiophores of this fungus were very fragile and easily disrupted, the fungus was subjected to environmental SEM, which retains the sporophores intact (spores attached), and images were recorded with a Philips XL 30 ESEM FEG (Philips, IL, USA; see Figure 1). A gaseous secondary electron detector was applied with a spot size of 3 at $15 \mathrm{kV}$. The temperature was $4{ }^{\circ} \mathrm{C}$, with a chamber pressure that ranged from 5 to 6 Torr, providing humidity up to $100 \%$ around the sample. Identification of endophytic Periconia sp. was performed with the help of the characteristic features and standard taxonomic manuals. ${ }^{36-41}$

\section{Fermentation, extraction and characterization of fungal piperine} The piperine was isolated from 4-week-old shake cultures of endophytic Periconia sp. grown in 21 of potato dextrose broth flasks at $25 \pm 2{ }^{\circ} \mathrm{C}$. The fungal biomass was removed from flasks by filtration and the culture fluid was extracted twice with equal volumes of ethyl acetate. The ethyl acetate fraction was dried by flash evaporation and the yield of crude material was up to $5.4 \mathrm{~g}$. This material was dissolved in a minimal volume of chloroform and was subjected to silica gel (size 60-120 mesh, Ranbaxy, Bangalore, India) column chromatography on a $3 \times 30-\mathrm{cm}$ column. The organic solvent extract was applied to the column and eluted using $500 \mathrm{ml}$ of ethyl acetate/methanol/water (10:1:0.1 (v/v)). The first $100 \mathrm{ml}$ eluted from the column was discarded and the next $400 \mathrm{ml}$ eluted from the column (possessing bioactivity) was taken to dryness ( $1.3 \mathrm{~g}$ ), redissolved in $2.0 \mathrm{ml}$ of chloroform, and placed on a second silica gel column (identical in size to the first column). It was eluted with $n$-hexene/ethyl-acetate/methanol/water $(2: 3: 2: 3(\mathrm{v} / \mathrm{v}))$. Fractions $(20 \mathrm{ml})$ were collected and the bioactivity appeared at 90-120 ml. The residue was dissolved in a minimum quantity of chloroform. Adding diethyl ether to this solution resulted in immediate appearance
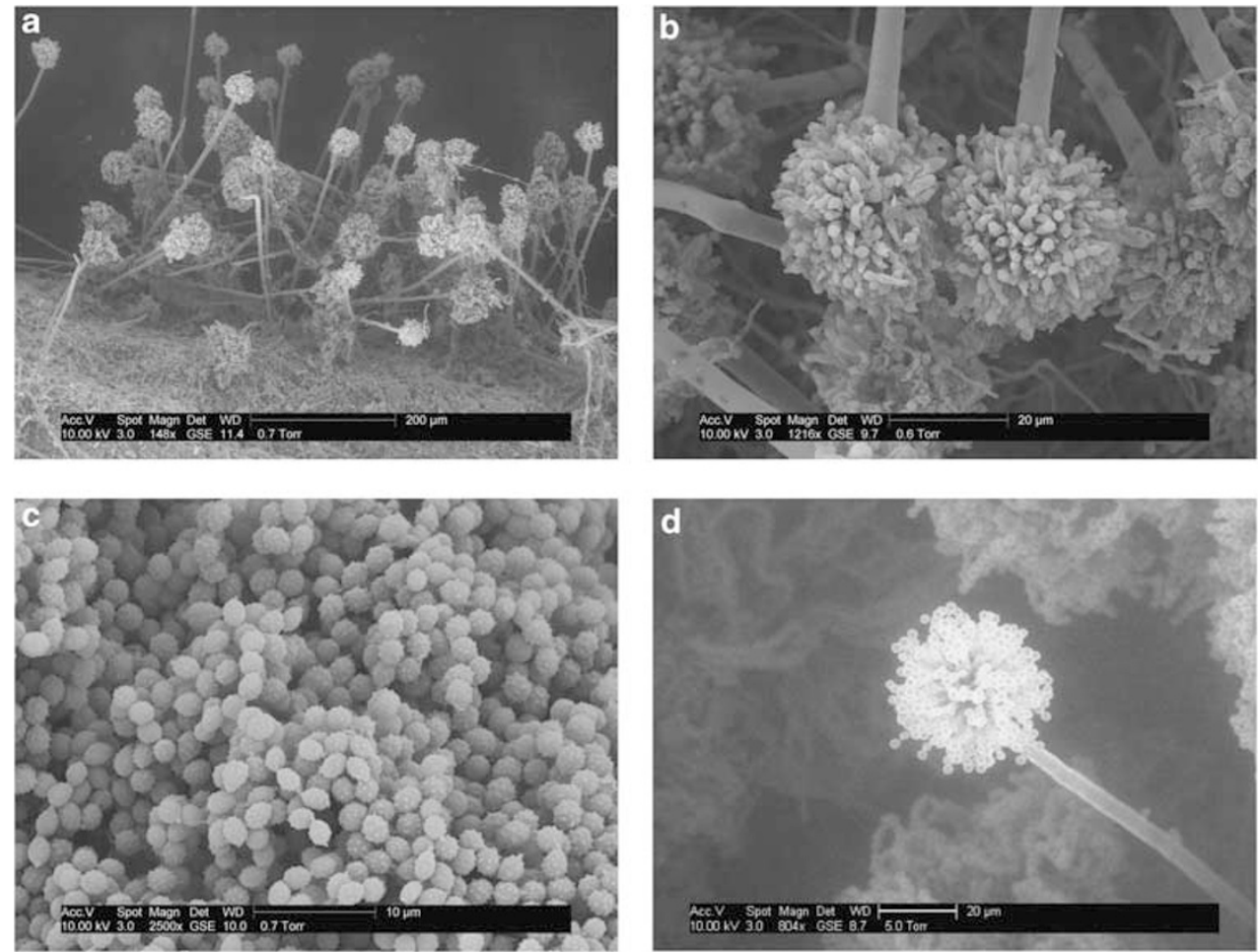

Figure 1 SEM image of Periconia sp. showing conidiophores and hyphae (a) and conidiophores with attached conidiogenous cells (b), warty conidia (c) and single conidiophore with an assemblage of spores (s) as taken by environmental SEM (d). Magnifications are shown by the bars on each illustration. 
and separation of needle-shaped crystals of piperine (yield $\sim 750 \mathrm{mg}$ ). The compound was purified by repeated crystallization to obtain shining yellow needles of piperine. The thin-layer chromatogram of the isolated compound showed a single peak at $R_{\mathrm{f}} 0.33$ and its UV absorption spectrum exactly overlapped that of the standard piperine. Furthermore, detection of piperine was made by its fluorescence at $340 \mathrm{~nm}$ and by spraying the plate with vanillin sulfuric acid spray reagent followed by gentle heating, resulting in a yellowish color spot. ${ }^{42}$ The calculated best-fit empirical formula of this fungal compound 'piperine' is $\mathrm{C}_{17} \mathrm{H}_{19} \mathrm{~N} \mathrm{O}_{3}$ (5-(3,4-Methylenedioxyphenyl)-1-piperidinopent2,4-dien-1-one) and the calculated MW was $285.33 \mathrm{~g} \mathrm{~mol}^{-1}$.

\section{Single-crystal X-ray crystallography of piperine}

The purified extract when dissolved in minimum chloroform, and by adding diethyl ether to this solution, resulted in the formation of numerous small needle-shaped yellowish crystals of piperine. Using a nylon loop, a suitable specimen (crystal of size $0.60 \times 0.50 \times 0.40 \mathrm{~mm}^{3}$ ) was chosen and mounted on a Bruker X8 APEX II diffractometer (Bruker AXS, Madison, WI, USA) $(\mathrm{MoK} \alpha$ radiation) and cooled to $-100^{\circ} \mathrm{C}$. Data collection and reduction were done using Bruker APEX2 (v. 1.0-22 user manual, Bruker AXS, 2004) and SAINT + (v. 6.02 user manual, Bruker AXS, 1999) software packages. An empirical absorption correction was applied with SADABS (GM Scheldrick, Program for Empirical Absorption Correction of Area Detector Data, University of Göttingen, 1996). Structures were solved by direct methods and refined on F2 by fullmatrix least-squares techniques using the SHELXTL (G.M. Scheldrick, v. 5.10 Bruker AXS, 1999d) software package. All non-hydrogen atoms were refined anisotropically. Hydrogen atoms were found in a difference fourier map and refined isotropically. Overall, 15940 reflections were collected, 4430 of which were symmetry independent $R_{\text {(int) }}=0.0275$; and final $\mathrm{R}$ factor was $4.13 \%$ with 3305 'strong' reflections (Fo $>4 \sigma(\mathrm{Fo})$ ). The crystal belongs to monoclinic space group P 2(1) $/ n$, with $a=8.6712(6), b=13.4428(8)$ and $c=12.9744(9) \AA$. The molecular structure was determined by single-crystal X-ray diffraction (see Figure 2 and supplementary data).

\section{Antimycobacterial bioassay}

Bioassays using the disc diffusion method were performed against two different strains of mycobacteria, a clinical multidrug resistant strain of Mycobacterium tuberculosis and an atypical mycobacterium, M. smegmetis. A uniform lawn for each of strains was prepared by streaking on molten middle brook B7H11 agar medium (Himedia, Mumbai, India) for Mycobacterium in fresh sterilized petri
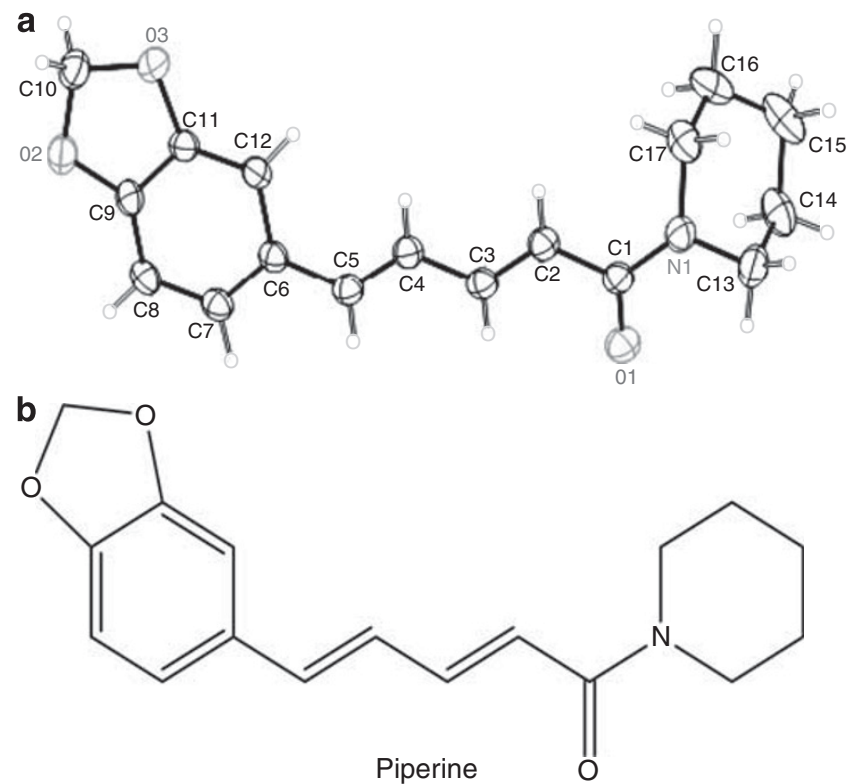

Figure 2 Single-crystal structure of piperine (a), and the molecular structural formula (b) on the basis of single-crystal X-ray crystallography. A full color version of this figure is available at The Journal of Antibiotics journal online. plates ( $9 \mathrm{~mm}$, Borosil, Bonn, Germany). An aliquot of $2.0 \mathrm{ml}$ fungal piperine of four different concentrations $\left(2,5,10,15 \mathrm{mg} \mathrm{ml}^{-1}\right)$ was placed onto a sterile disc and the solvent (that is, chloroform) allowed to evaporate to dryness on the surface in a decontaminated laminar hood for $15 \mathrm{~min}$. The zone of inhibition surrounding the disc on a bacterial plate was recorded in three replicates by incubating the plates of $M$. tuberculosis for 20 days and those of M. smegmetis for 5-7 days. Microplate alamar blue bioassay was performed with the purified fungal piperine to determine the MICs. A culture suspension of test strains was diluted upto $10^{5} \mathrm{cfu} \mathrm{m}^{-1}$ in 1:25 MB7H9 GC broth supplemented with glycerol and casitone. To determine the MIC values, different piperine concentrations were prepared from 0.05 to $6 \mathrm{mg} \mathrm{ml}^{-1}$. $100 \mu \mathrm{l}$ test strains of $M$. tuberculosis and M. smegmetis inoculums were added into a 96-well microtiter plate. After incubation for about 7 days, $50 \mu$ l of freshly prepared 1:1 mixture of alamar blue reagent and $10 \%$ tween 80 were added to each control well and incubated at $37{ }^{\circ} \mathrm{C} .{ }^{43}$ If the well turned pink, indicating growth, the reagent mixture was added to all wells. The MIC was defined as the lowest sample concentration that prevented a color change from blue to pink.

\section{Statistical analysis and data reduction}

The inhibition experiments were performed using plates as replicates and values given are means of these. Data were tested for normality, and a onefactor analysis of variance was conducted to determine whether the amount of inhibition differed between piperine concentration treatments. Means were separated by the Tukey HSD test.

\section{RESULTS AND DISCUSSION}

A total of 42 isolates of filamentous fungi, 17 isolates of bacteria and 5 actinomycetes were isolated and identified as microbial endophytes residing in the living tissues of $P$. longum plants. Among the dominant fungal isolates were species of Pestalotiopsis, Fusarium, Colletotrichum, Arthobotrys, Arthrinium, Cladosporium and Aspergillus, whereas among bacteria and actinomycetes, dominant isolates recovered were species of Streptomyces, Burkholderia, Pseudomonas, Nocardiopsis, Bacillus, Acetobacter, Enterobacter, Serratia and Paenibacillus, etc. (unpublished data, manuscript in preparation). After isolating endophytic microbes from $P$. longum plant, we initiated a comprehensive screening program to find potential endophytes having activity against Mycobacterium spp. in a disc diffusion agar bioassay, and those that did were selected for further study to identify and characterize the bioactive compounds. One isolate of endophytic Periconia sp. showed very significant activity against Mycobacterium spp. This particular strain was isolated from leaf tissues of $P$. longum and was recorded as an incidental rare strain ( $1 / 42$ isolates). Members of Periconia have a very wide host range as an endophyte and have been reported from several other pharmaceutically important plants, such as Azadirachta indica, ${ }^{33}$ Eucalyptus citriodora, ${ }^{44}$ Torriya grandifolia etc. ${ }^{45}$ The SEM study of this strain showed very prominent characteristic long conidiophores, dark, long, stout and shortly branched near the apex, bearing attached groups of conidiogenous cells at their tips (Figures la and $b$ ). As the chains of conidia are very fragile, only a few conidia are visibly attached on conidiogenous cells (Figure $1 \mathrm{~b}$ ), which is why the environmental SEM was performed to scan the head of conidiophores with attached conidia (Figure 1d). The individual conidia were warty (Figure 1c) and commonly bore a tubular segment of the conidiophores attached to them. This isolated Periconia sp. was deposited in the fungal type culture collection (f-TCC), Banaras Hindu University, India (f-TCCPERI-2894). A biologically active fraction with very significant antimycobacterial activity was screened and identified as 'piperine', a well-known alkaloid of the pepper plant and the chemical responsible for the pungency of the pepper spices. This was surprising as the only source known for this alkaloid is the host plant itself, but this is the first report of this compound being produced by an 

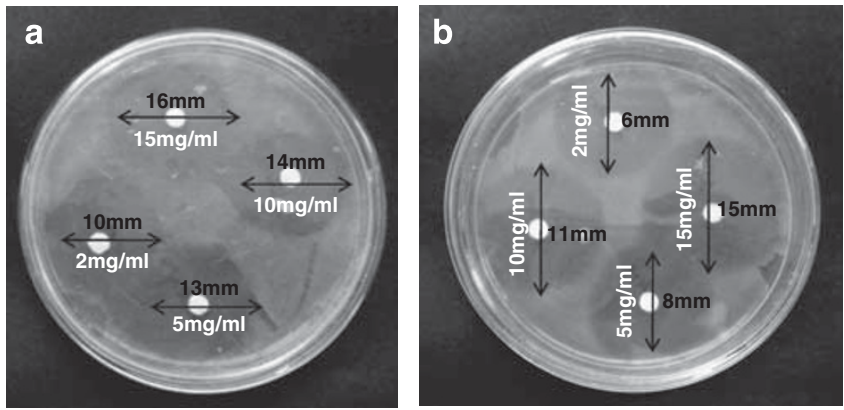

Figure 3 The disc diffusion bioassay against the Mycobacterium tuberculosis (a) and $M$. smegmetis (b). Four different concentrations (2, 5, 10, $15 \mathrm{mg} \mathrm{ml}^{-1}$ ) of purified fungal piperine show different activity and showed that as the concentration increased, the inhibition zones were also widened.

endophytic fungus from the pepper plant. The piperine is an alkaloid and a piperidine derivative of the vanilloid family of compounds and is weakly basic. It is essentially tasteless at first but has a burning aftertaste. The compound is believed to have the ability to enhance activity for some nutrients and drugs and has alleged anti-inflammatory capability and several other pharmacological activities. ${ }^{7}$ Additionally, there are several other reports available that confirm the activity of piperine against Mycobacterium spp.; such as its role in inhibition of Rv1258c, a putative multidrug efflux pump of M. tuberculosis. ${ }^{46}$ The piperine we obtained from the endophytic Periconia sp. was naturally functional and the spectroscopic study of this compound revealed $99 \%$ similarity with piperine from the host itself. Although the yield of the purified piperine is very low $(\sim 750 \mathrm{mg})$, when dissolved in a mixture of chloroform/diethyl ether, the yellowish needle-shaped crystals were obtained. Often, crystals were formed only when the extracts were highly purified and no additional components were mixed with them. These crystals were then subjected to single-crystal X-ray crystallography (Figure 2a), which were in close proximity to the natural/parent piperine from the host plant. The molecular structure was obtained with the details of parameters as obtained from crystallography (Figure $2 b$, supplementary data). This fungal piperine shows very promising potential as an antimycobacterial agent as it showed prominent growth inhibitory activity against two strains of Mycobacterium spp. (Figure 3). In M. tuberculosis, all treatments differed from each other and there was a clear trend of increasing inhibition with increased fungal piperine concentration $\left(F_{3,8}=248.3, P<0.001\right)$. A very similar pattern was seen with $M$. smegmetis $\left(F_{3,8}=452.6\right.$, $P<0.001)$. In general, stronger inhibition was observed with M. tuberculosis (Figure 4a) than with M. smegmetis (Figure 4b). This general trend was also confirmed by the determination of MICs against these two test strains that ranged from 1.74 for $M$. tuberculosis to $2.62 \mu \mathrm{g} \mathrm{m} \mathrm{m}^{-1}$ for M. smegmetis (Figure 3). The mycobacterial bioassay established by us and the MICs by the alamar blue assay provided a very clear indication of antimycobacterial activity with purified fungal piperine. These bioactivities confirm that the fungal piperine is as active and functional as piperine from the host plant.

Periconia spp. isolated from Torriya grandifolia was also reported to produce taxol, ${ }^{45}$ thus it seems that member of this genus would be well worth considering in 'bio-prospecting' for novel pharmaceuticals. However, the ability of endophytes to produce these chemicals raises several intriguing questions that have yet to be answered, including, whether the compound is first synthesized by the plant or by the fungus and whether there is a transfer of genetic information between the two. Furthermore, we need to determine whether microbes are

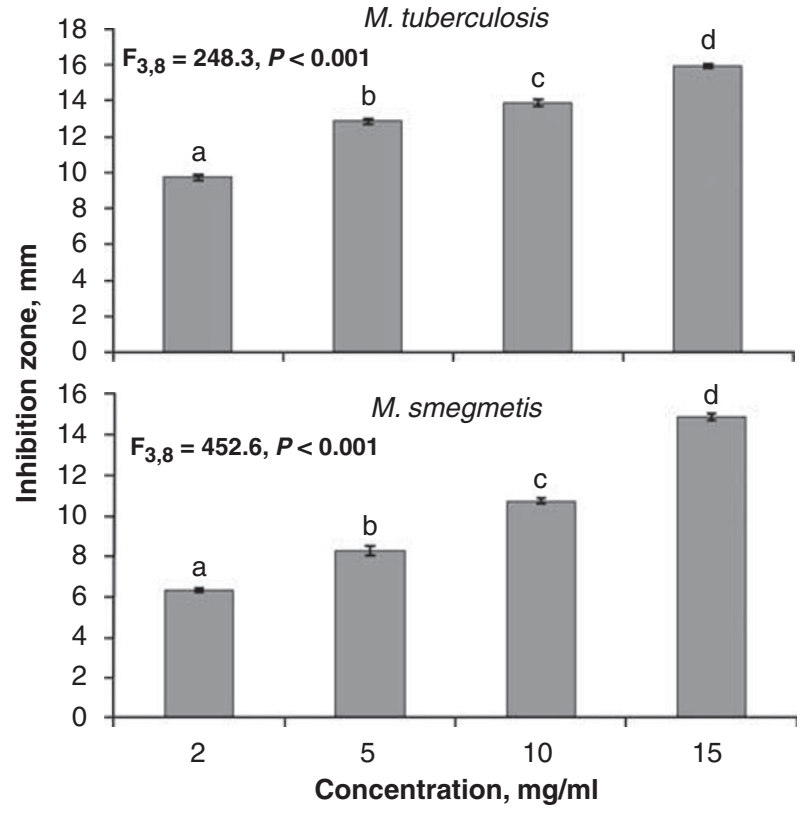

Figure 4 The inhibition pattern of the two strains caused by different concentrations of piperine.

capable of communicating with each other within the plant and whether chemical production occurs as a result of interaction between microbes. It remains a question of further research as to how and why this particular strain of Periconia sp. produces an alkaloid identical to that derived from its host plant. As suggested by Tan and Zou, ${ }^{25}$ there may be the possibility of genetic recombination between endophyte and the host in the course of evolutionary symbiosis, and if this happens, then the exact mechanism should be resolved urgently.

The potential of endophytic microbes in synthesizing bioactive compounds within their host plants is a very significant aspect of the host endophyte interaction. ${ }^{1,29,47-49}$ The discovery of fungal endophytes with piperine-producing potential may have significant scientific and industrial applications. However, there are several factors associated in its industrial implications; one major factor is the metabolic regulation of fungus in axenic culture. It is observed that after second or third successive generations, this potential was substantially attenuated, and the production of interested molecule dropped below the limit of detection. ${ }^{6}$ This might be assumed due to lack of host stimuli in axenic cultures, or rapid inactivation or transformation of the interested molecule. Meanwhile, if endophytes can produce the same rare and important bioactive compounds as their host plants, this would not only reduce the need to harvest slowgrowing and possibly rare plants, but also help to preserve the world's ever-diminishing biodiversity. Furthermore, it is recognized that a microbial source of a high-value product may be easier and more economical to produce effectively, thereby reducing its market price.

Thus, in summary, our phytochemical findings provide a basis for exploiting endophytic Periconia sp. as a new alternative source for piperine and other related analogs from $P$. longum plants to meet the growing challenge of drug resistance against mycobacteria and other potent human pathogens. We may suggest that these results could be exploited to use piperine alone and/or in combination with other available drugs in an attempt to overcome drug resistance in Mycobacterium spp. specifically. 


\section{ACKNOWLEDGEMENTS}

We wish to extend our thanks to the Professor-in charge, centre of experimental medicine and surgery, Institute of Medical Sciences, Banaras Hindu University, Varanasi, India. The financial assistance to VCV from the Council of Scientific and Industrial Research (CSIR-09/013(205)/2008/EMR-I, dt.24-09-2008) New Delhi is also gratefully acknowledged.

1 Stierle, A., Strobel, G. A. \& Stierle, D. Taxol and Taxane production by Taxomyces andreanae an endophytic fungus of Pacific yew. Science 260, 214-216 (1993).

2 Schulz, B., Boyle, C., Draeger, S., Rommert, A. K. \& Krohn, K. Endophytic fungi: a source of novel biologically active secondary metabolites. Mycol. Res. 106, 996-1004 (2002).

3 Kusari, S., Lamshöft, M., Zühlke, S. \& Spiteller, M. An endophytic fungus from Hypericum perforatum that produces hypericin. J. Nat. Prod. 71, 159-162 (2008).

4 Zhang, L. B., Gou, L. H. \& Zeng, S. V. Preliminary study on the isolation of endophytic fungus of Catharanthus roseus and its fermentation to produce product of therapeutic value. Chin. Trad. Herb. Drugs 11, 805-807 (2000).

5 Tung, C. Y., Yang, D. B. \& Gou, M. A Preliminary study on the condition of the culture an isolate of endophytic fungus producing vincristine. J. Chuxiong Norm. Univ. 6, 39-41 (2002).

6 Kusari, S., Zülke, S. \& Spiteller, M. An endophytic fungus from Camptotheca acuminata that produces camptothecin and analogues. J. Nat. Prod. 72, 2-7 (2009).

7 Pie, Y. Q. A review of pharmacology and clinical use of piperine and its derivatives. Epilepsia 24, 177-183 (1983).

8 Srinivasan, K. Black pepper and its pungent principle-piperine: a review of diverse physiological effects. Crit. Rev. Food Sci. Nutr. 47, 735-748 (2007).

9 Li, S., Wang, C., Wang, M., Li, W., Matsumoto, K. \& Tang, Y. Antidepressant like effects of piperine in chronic mild stress treated mice and its possible mechanisms. Life Sci. 80, 1373-1381 (2007).

10 Chonpathompikunlert, P., Wattanathorn, J. \& Muchimapura, S. Piperine, the main alkaloid of Thai black pepper, protects against neurodegeneration and cognitive impairment in animal model of cognitive deficit like condition of Alzheimer's disease. Food Chem. Toxicol. 48, 798-802 (2010).

11 Fu, M., Sun, Z. H. \& Zuo, H. C. Neuroprotective effect of piperine on primarily cultured hippocampal neurons. Biol. Pharm. Bull. 33, 598-603 (2010).

12 Lee, E. B., Shin, K. H. \& Woo, W. S. Pharmacological study on piperine. Arch. Pharmacol. Res. 7, 127-132 (1984).

13 Bae, G. S. et al. Inhibition of lipopolysaccharide-induced inflammatory responses by piperine. Eur. J. Pharmacol. 642, 154-162 (2010).

14 Khajuria, A., Thusus, N., Zutshi, U. \& Bedi, K. L. Antioxidant potential of piperine on oxidant induced alterations in rat intestinal lumen. Indian Drugs 34, 557-563 (1997).

15 Prakash, U. N. \& Srinivasan, K. Gastrointestinal protective effect of dietary spices during ethanol-induced oxidant stress in experimental rats. Appl. Physiol. Nutr. Metab. 35, 134-141 (2010).

16 Bezerra, D. P. et al. In vitro and in vivo antitumor effect of 5-FU combined with piplartine and piperine. J. Appl. Toxicol. 28, 156-163 (2008).

17 Koul, I. B. \& Kapil, A. Evaluation of the liver protective potential of piperine, an active principle of black and long peppers. Planta Med. 59, 413-417 (1993).

18 Takumi, I., Mizuho, I., Siho, T., Yuzi, I. \& Hideyuki, Y. Piperine, a pepper ingredient, improves the hepatic increase in free fatty acids caused by $2,3,7,8$ - tetra chlorodibenzo- $p$-dioxine. J. Health Sci. 54, 551-558 (2008).

19 Chandrashekhar, R. B., Jithan, A., Narsimha, R. Y. \& Malla, R. V. Fabrication and investigations on hepatoprotective activity of sustained release biodegradable piperine microspheres. Int. J. Phar. Sci. Nano Tech. 1, 87-96 (2008).

20 Timmers, L. Herbal Medicines Used against Epilepsy in Developing Countries Publication number PUG/94-4; (Publicaties Wetenschapswinkel Geneesmiddelen: Vrouwen, The Netherlands, 1994).

21 Hu, R. Q. \& Davies, J. A. Effects of Piper nigrum L. on epileptiform activity in cortical wedges prepared from DBA/2 mice. Phytother. Res. 11, 222-225 (1997).

22 Karan, R. S., Bhargava, V. K. \& Garg, S. K. Effect of piperine on the pharmacokinetic profile of isoniazid in rabbits. Indian J. Pharmacol. 30, 254-256 (1988).

23 Zutshi, R. K., Singh, R., Zutshi, U., Johri, R. K. \& Atal, C. K. Influence of piperine on rifampicin blood levels in patients of pulmonary tuberculosis. J. Assoc. Phys. India 33, 223-224 (1985).
24 Bano, G., Amla, V., Raina, R. K., Zutshi, U. \& Chopra, C. L. The effect of piperine on pharmacokinetics of phenytoin in healthy volunteers. Planta Med. 53, 568-569 (1987).

25 Tan, R. X. \& Zou, W. X. Endophytes: a rich source of functional metabolites. Nat. Prod. Rep. 18, 448-459 (2001).

26 Strobel, G. A. Microbial gifts from the rain forest. Can. J. Phtopathol. 24, 14-20 (2002).

27 Strobel, G A., Daisy, B., Castillo, U. \& Harper, J. Natural products from endophytic fungi. J. Nat. Prod. 67, 257-268 (2004).

28 Gunatilaka, A. A. L. Natural products from plant-associated microorganisms: distribution, structural diversity, bioactivity and implications of their occurrence. J. Nat. Prod. 69, 509-526 (2006).

29 Guo, B., Wang, Y., Sun, X. \& Tang, K. Bioactive natural products from endophytes: a review. Appl. Biochem. Microbiol. 44, 136-142 (2008).

30 Verma, V. C., Kharwar, R. N. \& Strobel, G. A. Chemical and functional diversity of natural products from plant associated endophytic fungi. Nat. Prod. Comm. 4, 1511-1532 (2009a).

31 Aravind, R., Kumar, A., Eapen, S. J. \& Ramana, K. V. Endophytic bacterial flora in root and stem tissues of black pepper (Piper nigrum L.) genotype: isolation, identification and evaluation against Phytophthora capsici. Lett. Appl. Microbiol. 48, 58-64 (2009).

32 Dahiya, J. S., Woods, D. L. \& Tiwari, J. P. Piperine from an Ulocladium sp. Phytochemistry 27, 2366 (1988)

33 Verma, V. C., Gond, S. K. Kumar, A. Kharwar, R. N. \& Strobel, G. A. Endophytic mycoflora of bark, leaf, and stem tissues of Azadirachta indica A. Juss. (Neem) from Varanasi (India). Microb. Ecol. 54, 119-125 (2007).

34 Verma, V. C., Gond, S. K., Kumar, A., Mishra, A., Kharwar, R. N. \& Gange, A. C. Endophytic actinomycetes from Azadirachta indica A. Juss.: isolation, diversity and anti-microbial activity. Microb. Ecol. 57, 749-756 (2009b).

35 Ezra, D., Hess, W. M. \& Strobel, G. A. New endophytic isolates of Muscodor albus, a volatile-antibiotic-producing fungus. Microbiol. 150, 4023-4031 (2004).

36 Barnett, H. L. \& Hunter, B. B. Illustrated Genera of Imperfect Fungi (4th ed) (Macmillan Publishers: New YorkCo ISBN: 0-89054-192-2 1998).

37 Ellis, M. B. Dematiaceous Hyphomycetes (Commonwealth Mycological Institute: Kew, Surrey, England, UK, 1971).

38 Ellis, M. B. More Dematiaceous Hyphomycetes (Commonwealth Mycological Institute: Kew, Surrey, England, UK, 1978).

39 Van Arx, J. A. The Genera of Fungi Sporulating in Pure Culture (AR Gantner Verlag KG: Vaduz, Liechtenstein, 1978)

40 Raper, K. B., \& Thom, C. A. Manual of the Penicillia (Elsevier Biomedical Press: Amsterdam, The Netherlands, 1949).

41 Ainsworth, G. C., Sparrow, F. K. \& Sussman, A. S. The Fungi-Advanced Treaties Vol. 4A (Academic Press: New York, USA, 1973).

42 Arshia, S., Marna, P. K., Paranjothy, K. L. K. \& Mohammad, A. Hepatoprotectant activity of alcoholic extract of Andrographis paniculata entrapped in sodium alginate micropallets. Nat. Remed. 7, 283-288 (2007).

43 Collins, L. \& Franzblau, S. G. Microplate alamar blue assay versus BACTEC 460 system for high-throughput screening of compounds against Mycobacterium tuberculosis and Mycobacterium avium. Antimicrob. Agents Chemother. 41, 1004-1009 (1997).

44 Kharwar, R. N., Gond, S. K., Kumar, A. \& Mishra, A. A comparative study of endophytic and epiphytic fungal association with leaf of Eucalyptus citriodora Hook. and their antimicrobial activity. World J. Microbiol. Biotechnol. 26, 1941-1948 (2010).

45 Li, Y., Sidhu, R. S., Ford, E., Hess, W. M. \& Strobel, G. A. The induction of taxol production in the endophytic fungus Periconia sp. from Torreya grandifolia. J. Indian Microbiol. 20, 259-264 (1993).

46 Sharma, S., Kumar, M., Sharma, S., Nargotra, A., Koul, S. \& Khan, I. A. Piperine as an inhibitor of Rv1258c, a putative multidrug efflux pump of Mycobacterium tuberculosis. J. Antimicrob. Chemother. 65, 1694-1701 (2010).

$47 \mathrm{Ji}$, Y., Bi, J. N., Yan, B. \& Zhu, X. D. Taxol-producing fungi: a new approach to industrial production of taxol. Chin. J. Biotechnol. 1, 1-6 (2006).

48 Gangadevi, V. \& Muthumary, J. Taxol, an anticancer drug produced by an endophytic fungus Bartalinia robillardoides Tassi, isolated from a medicinal plant, Aegle marmelos Correa ex Roxb. World J. Microbiol. Biotechnol. 24, 717-724 (2008).

49 Gangadevi, V. \& Muthumary, J. Taxol production by Pestalotiopsis terminaliae, an endophytic fungus of Terminalia arjuna (arjun tree). Biotech. Appl. Biochem. 52, 9-15 (2009)

Supplementary Information accompanies the paper on The Journal of Antibiotics website (http://www.nature.com/ja) 